\title{
Adaptation experience during the period following the transplantation in kidney transplant patients
}

\author{
Miim Kim', Sungok Chang ${ }^{2}$ \\ ${ }^{1}$ Department of Surgery-Transplantation, Korea University Anam Hospital, Seoul, Korea \\ ${ }^{2}$ College of Nursing, Korea University, Seoul, Korea
}

Background: Renal transplantation is a treatment that can be expected to improve the quality of life as well as the survival period compared to hemodialysis and peritoneal dialysis. Previous studies have shown variations in the rejection rate, survival rate, self-care performance, treatment order compliance, post-transplant management, immunosuppressive drug compliance, and competence sensitivity during the course of time following renal transplantation. Further, kidney transplant patients experienced fear, depression, anxiety, frustration, and helplessness after transplantation. Therefore, we set out to provide fundamental data that understand patients' lives in depth and establish effective nursing intervention strategies by comprehending the adaptation experience during the course of time following the transplantation.

Methods: This study conducted an in-depth interview using the interview questions prepared based on the adaptation theory of Roy (1970) to evaluate the adaptation experience during the course of time following the transplantation in kidney transplant patients, and performed quantitative research through topic analysis of Braun \& Clark (2006).

Results: The study included a total of 31 patients from a university hospital in Seoul who were under outpatient treatment after kidney transplantation (10 patients in 1 year or less after transplantation, 10 patients between 1 and 5 years, 11 patients in 5 years or more). The patient pool included those who were 20 years of age or older that understood the purpose of the study and voluntarily agreed to participate in the study. Data was collected from March, 2020 to April, 2021; individual in-depth interviews were conducted through semi-structured questions with field notes and recordings based on the adaptation theory of Roy (1970). The data collection was conducted in a quiet counseling room at the organ transplant center and the analysis was conducted using the transcript following the interview. The data analysis was performed using thematic analysis of Braun \& Clark (2006). It was shown that within a year after kidney transplantation, 'beginning of normalization' and 'taking on the role as a promoter of healthier' were evident and noticeable improvements were seen through 'expanding awareness of the world of living.' Between 1 and 5 years after kidney transplantation, 'role as a promoter of healthier' was stabilized through 'establishment of normalization' and 'increased self-esteem' was achieved through 'expanding network.' Patients in 5 or more years after kidney transplantation exhibited 'daily life with repeated management obsession and negligence' through 'comparing the lives of normal and abnormal people' and experienced 'unlimited expansion.' In conclusion, the patients with kidney transplantation showed differences in the experiences of normalization, promoter of healthier, and expansion during the course of time following the transplantation.

Conclusions: This study established the adaptation experiences during the course of time following the transplantation in kidney transplant patients and enabled detailed understanding of how their experiences vary. Based on the results of this study, it is necessary to recognize the shift in experience during the course of time following the transplantation in kidney transplant patients and to provide customized nursing care.

Corresponding author: Miim Kim

E-mail: kkimmi9251@korea.ac.kr

(C) The Korean Society for Transplantation

This is an Open Access article distributed under the terms of the Creative Commons Attribution Non-Commercial License (http://creativecommons.org/licenses/by-nc/4.0/) which permits unrestricted non-commercial use, distribution, and reproduction in any medium, provided the original work is properly cited. 